\title{
CRISIS CONVULSIVA COMO PRIMERA MANIFESTACIÓN CLÍNICA DE ESCLEROSIS MÚLTIPLE
}

Ramón Antonio Quintero Almenarez MD*, Manuel Guillermo Uribe Granja MD**, Paolo Solano Leal***

\section{Resumen}

La esclerosis múltiple es una enfermedad desmielinizante del sistema nervioso central cuyos hallazgos patológicos se encuentran principalmente en la sustancia blanca. Sin embargo, es paradójico que quienes padecen esta enfermedad tienen una probabilidad mayor que la población general de presentar crisis epilépticas.

Citamos a continuación el caso de un paciente joven con esclerosis múltiple tipo recaída remisión, que presentó una crisis como primera manifestación de su enfermedad. Los hallazgos imagenológicos de las lesiones corticales y yuxtacorticales encontrados se relacionan topográficamente con las manifestaciones clínicas de las convulsiones. Se analizan la epidemiología, clínica, fisiopatología, aproximación diagnóstica y terapéutica, así como el pronóstico a largo plazo de estos pacientes.

Palabras clave:: esclerosis múltiple, crisis epiléptica, epilepsia, resonancia magnética cerebral.

\section{Justificación}

Las crisis epilépticas pueden ocurrir como una manifestación clínica de la esclerosis múltiple, son debidas a lesiones desmielinizantes corticales o subcorticales, o a efectos adversos de medicamentos utilizados para tratar la esclerosis múltiple. Dichas crisis por presentarse con frecuencia como un epifenómeno durante la recaída, por lo regular no requieren tratamiento con fármacos anticonvulsivantes.

La presentación de este caso muestra que la esclerosis múltiple debe ser tenida en cuenta como una causa de epilepsia de novo en adultos jóvenes, al igual que nos enseña como el enfoque diagnóstico apropiado puede evitar el uso innecesario de anticonvulsivantes, el cual es un potencial generador de efectos adversos para el paciente.

Fecha recibido: I de diciembre 2005 - Fecha aceptado: 15 de diciembre 2005

* Residente de tercer año de neurología clínica, Fundación Universitaria de Ciencias de la Salud, Bogotá, D.C. Colombia.

** Neurólogo clínico, neurotoxicólogo, Hospital de San José, Bogotá, D.C. Profesor Asistente Fundación Universitaria de Ciencias de la Salud. (FUCS), Bogotá, D.C. Colombia.

* Estudiante de medicina Fundación Universitaria de Ciencias de la Salud. Bogotá, D.C. Colombia.

\section{Resumen de la historia clínica}

Hombre de 22 años admitido por cuadro clínico de tres días de evolución consistente en contracciones de aparición súbita en hemicara izquierda seguidas de movimientos clónicos del hemicuerpo ipsilateral, de dos minutos de duración con frecuencia de tres por día, asociados a dolor retroocular y disminución de la agudeza visual por el ojo izquierdo, debilidad $\mathrm{y}$ disestesias en miembros inferiores.

Antecedentes: hace diez años crisis focal motora con marcha jacksoniana tratada con carbamazepina. Dos episodios de neuritis óptica izquierda hace siete y dos años. Parálisis facial periférica izquierda hace 22 meses. Un episodio de déficit motor en hemicuerpo izquierdo hace 20 meses. En todas las ocasiones se manejó como recaídas de esclerosis múltiple y recibió pulsos de metilprednisolona por cinco días con mejoría.

Examen físico: el general es normal. En el neurológico se encontró en el ojo izquierdo agudeza visual 20/50, desaturación al rojo y azul, fenómeno pupilar 
de Marcus Gunn. Fuerza de 4/5 en miembros inferiores e hiperrreflexia rotuliana y aquiliana simétrica. Nivel sensitivo T10. Resto normal.

Se consideraron como posibilidades diagnósticas:

1. Esclerosis múltiple tipo recaída-remisión.

2. Mielitis transversa secundaria a la anterior.

3. Neuritis óptica secundaria a la primera.

4. Epilepsia focal sintomática.

En el hospital se practicó estudio de resonancia magnética con contraste (RMC) los hallazgos se observan en la Figura 1. El electroencefalograma (EEG) no muestra descargas epilépticas. Se administraron pulsos de metilprednisolona durante cinco días con mejoría de la agudeza visual a 20/40, resolución total de los síntomas sensitivos, del dolor ocular y de la debilidad. Se suspendió la carbamazepina sin recurrencia de crisis.

\section{Comentarios}

La aparición de crisis epilépticas es entre tres a seis veces mayor en pacientes con esclerosis múltiple (EM) que en la población general, tienen una prevalencia entre 1 y $5 \%{ }^{1,2,3}$. La EM debe tenerse en cuenta como causa de epilepsia de novo en adultos jóvenes. Las crisis pueden ocurrir en cualquier momento de la evolución de la enfermedad o ser su única manifestación. Hasta un $5 \%$ de los pacientes pueden presentar crisis de uno a cinco años antes de otra manifestación de (EM) ${ }^{4}$, aunque algunos estudios muestran porcentajes hasta del $48 \%$ de los $\operatorname{casos}^{2}$, las crisis también pueden ocurrir como manifestación única durante la recaída o estar asociadas a otros signos clínicos o radiológicos relacionados con la recaída de $\mathrm{EM}^{4,5}$.

El tipo de crisis es variable de un paciente a otro y se encuentra relacionado con la localización de la lesión cerebral, la descrita con mayor frecuencia es la tónico clónica generalizada; otros tipos de crisis se observan en la Tabla $1^{2,6,7,8}$. Se han descrito casos de epilepsia del lóbulo temporal corroborados con lesiones desmielinizantes en RMC y descargas en el EEG en dicha localización ${ }^{9}$.

En un paciente con EM con historia de ilusiones visuales se observaron paroxismos epilépticos occipitales en el EEG. Un caso de epilepsia refleja un tipo musicogénica también fue descrito ${ }^{2}$. Es poco frecuente el status epiléptico.

Las crisis se atribuyen a lesiones desmielinizantes corticales o yuxtacorticales, pueden estar relacionadas con edema perilesional deberse a los medicamentos utilizados en el tratamiento de EM; algunos estudios sugieren que el interferón beta reduce el umbral convulsivo; el uso de baclofeno en altas dosis así como la suspensión abrupta de éste, son potenciales generadores de crisis; el empleo de 4-aminopiridina en el tratamiento de EM también se ha mencionado como responsable de convulsiones. Para realizar el diagnóstico de crisis es importante practicar una RMC con contraste, con el fin de localizar las lesiones epileptogénicas ${ }^{10,11,12}$. Son de ayuda también los potenciales evocados ${ }^{11}$. El EEG en estos pacientes es importante para correlacionar la localización de lesiones desmielinizantes en las imágenes y sitios de descargas epileptiformes; sin embargo en estudios realizados el EEG puede ser normal en el 25 a $50 \%$ de los pacientes ${ }^{2,6}$.

El pronóstico de las crisis no está claramente relacionado con la severidad de la EM; a largo plazo es favorable, en especial cuando las convulsiones solo se presentan durante la recaída y por lo regular no se requieren fármacos antiepilépticos. En algunos casos de status epiléptico se encuentra buena respuesta a esteroides endovenosos cuando se asocia con recaídas 2,3 . El tamaño y la localización de la lesión son los factores determinantes en el desarrollo de epilepsia crónica. Los medicamentos antiepilépticos han demostrado éxito en el control de la gran mayoría de crisis, como es el caso de la carbamazepina a dosis de $400 \mathrm{mg} /$ día, lo mismo el ácido valproico y la fenitoína ${ }^{2}$. Los tipos de EM 
asociados con crisis son recaída-remisión $(85 \%)$ y secundaria progresiva $(15 \%)^{4}$.

Nuestro paciente presenta más de dos ataques y evidencia clínica objetiva de las lesiones, lo que hace el diagnóstico de esclerosis múltiple (EM). Ha presentado dos crisis motores con marcha jaksoniana de iguales características, la primera tres años antes de otras manifestaciones de EM. En la RMC se evidencia una lesión hiperintensa en el T2, frontotemporal derecha de localización cortico-subcortical, la

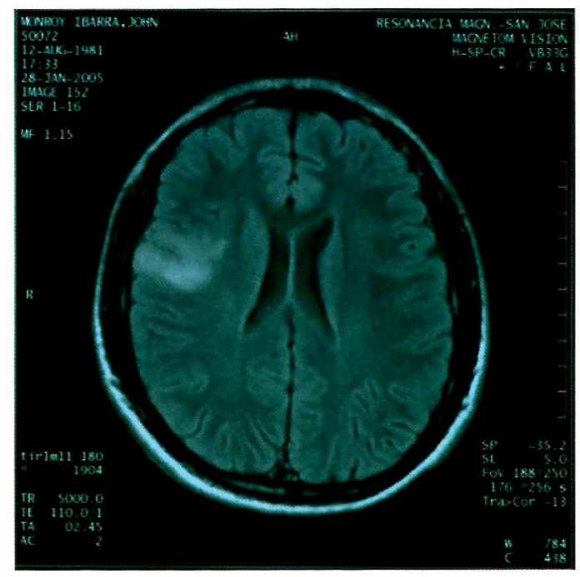

Figura I. IRMT2 Lesión hiperintensa córtico-subcortical derecha en la resonancia magnética cerebral en T2.

Tabla I. Tipos de crisis usualmente relacionadas con esclerosis múltiple

Tónico clónica generalizada

Motora con marcha jacksoniana

Motora sin marcha jacksoniana

Afásica

Disfásica

Musicogénica

Visuales cual tiene correlación clinica con las convulsiones. Consederamos que las crisis son secundarias a dicha lesión, en la primera ocasión correspondió a la primera manifestación de EM y en la segunda hizo parte de la recaída actual.

\section{Referencias}

1. Poser CM,Brinar VV.Epilepsy and multiple sclerosis. Epilepsy Behav 2003 Feb;4(1):6-12

2. Spatt J,Chaix R,Mamoli B.Epilectic and no epileptic seizures in multiple sclerosis.J Neurol 2001; 248:49

3. Kinnunen E,Wikstrom J.Prevalence and prognosis of epilepsy in patients with multiple sclerosis.Epilepsia 1986 Nov-Dec;27(6):729-33

4. Sokic D,Stojsavljevic N,Drulovic Jelena, et al.Seizures in multiple sclerosis.Epilepsia 2001; 42 (1 ):72-79

5. Garcia-Asencio S,Lopez del Val J,Barrerna R, et al.Epilepsy as the first sign of multiple sclerosis.Rev Neurol.1997 Jan ;25(137):80-3

6. Nyquist PA, Cascino GD, Rodríguez M. Seizures in patients with multiple sclerosis seen ay Mayo Clinic, Rochester, Minn, 1990-1998. Mayo Clinic Proc 2001 oct. 76 (10): 983-6

7. Lacour De S. J. Revenco E, et al. Acute aplasia in multiple sclerosis: A multicenter study of 22 patientes. Neurology 2004 mar 23; 62 (6):974-7

8. Spatt J. GoldenbergG, Mamoli B. Simple dysphasic seizures as the sole manifestation of relapse in multiple sclerosis. Epilepsia 1994 nod-dec; 35 (6): 1342-5

9. Gambardella A. Valentino P. Labate A, et al. Temporal lobe epilepsy as a unique manifestarion of multiple sclerosis. Can J Neurol Sci. 2003 aug; 30 (3): 228-32

10. Truyen L. BarkhofF, Frequin ST, et al. Magnetic resonance imaging of epilepsy in multiple sclerosis: a case control study. Implications for treatament trials with 4-aminopiridine. Mult Scler 1996 feb; 1 (4): 213-7

11. Murillo MJ, Aladro Y. Mompeo B. Epileptic crisis and multiple sclerosis: anatomo-clinical correlation. Rev.Neurol 1999, sept. 29 (6): 508-10

12. Thompson AJ, Kermode AG, Moseley IF. Seizures due to multiple sclerosis seven patients with MRI correlations. J Neurol Neurosurg Psychiatry, 1993 dec. 56 (12): 1317-20
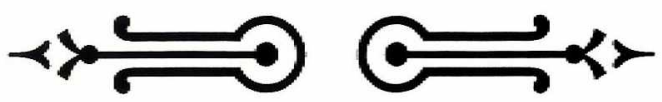